\title{
Uchronie: Revisiting Analogue Computing to Expand the Language of A/V Synthesis
}

\author{
Mat Dalgleish \\ Faculty of Arts \\ University of Wolverhampton, UK \\ m.dalgleish2@wlv.ac.uk
}

\author{
Chris Payne \\ Faculty of Arts \\ University of Wolverhampton, UK \\ c.c.payne@wlv.ac.uk
}

\author{
Richard Burn \\ Faculty of Arts \\ University of Wolverhampton, UK \\ rburn97@gmail.com
}

\begin{abstract}
Compared to acoustic instruments, the evolution of the synthesizer has been rapid but fragmented. With reference to the theory of Chion, Uchronie explores the audiovisual possibilities of borrowing from general-purpose analogue computers. These were briefly explored in the 1960s, but have since been neglected in favour of more conventional musical processes and associated language.
\end{abstract}

Audiovisual. Analogue computing. Chion. Modular synthesizer. Eurorack.

\section{INTRODUCTION}

According to Owen (2009), the first confirmed musical instrument appeared around 40,000 years ago and is described as a flute carved from a vulture bone. With a world population of around 10 million, people were distributed across the world in small groups and thus early innovation was inevitably a slow process. It appears that the accelerated development of musical instruments (and other technologies) began to happen only as people began to congregate in greater numbers approximately 5000 years ago, and may have been enabled by the sharing of ideas amongst large numbers of individuals living in close proximity. For Johnson (2011), the establishment of the dense populations found in true cities fostered new paradigms of invention. In particular, he suggests that cities facilitated the recording and preservation of ideas, thereby providing the basis for incremental innovation (i.e. a series of small improvements made to a technology over time). Incremental innovation is common in the acoustic musical instrument domain: most of the canonical acoustic instruments seen today have evolved gradually over hundreds or sometimes even thousands of years (Paradiso, 1998).

The modern day piano for instance is rooted in the invention of the organ by Ctesibius in the third century BC (Bicknell, 1996). While the process of moving a column of air by blowing was first employed much earlier, Ctesibius' Hydraulis took this idea and furthered it using newly available technology (McKinnon, 2014). In particular, he developed a water-driven mechanical device to stabilise the wind pressure produced by bellows pumping air through a set of musical pipes. These pipes were then manipulated or played using flaps that formed a rudimentary keyboard (or organ manual). Meeus (2014) notes that the keyboard is not in itself the instrument, but instead only linked to the means of producing sound. This distinction is vitally important, for it implies the potential to separate the performance interface from the means of sound generation. In the acoustic domain the two elements required a mechanical connection: the interface had to physically act upon and stimulate the sound generation mechanism. Nevertheless, it was possible to transfer the performance interface from one instrument to another. Thus, by the early 14th century the organ keyboard had been applied to the plucked strings of the clavichord, and, by the end of the century, the harpsichord.

Harpsichords pluck their strings invariably when their keys are pressed. Thus, one factor driving the development of the piano was a desire to create a harpsichord that was sensitive to intensity of sound. The piano does this by varying the speed at which its hammers strike its strings: the harder a key is pressed the higher the velocity of the associated impact between hammer and string. By the 18th century the piano (and its keyboard) had started to become ubiquitous. In its dual role as an aspirational concert instrument and a form of home entertainment, it became embedded not only in the fabric of Western music but also in popular culture. If the evolution of the piano and other "canonical" instruments has stalled in the last century, perhaps becoming subject to what Risset (1978) calls "sociological weight," electronic instruments have 
evolved rapidly over the same period, sometimes in unexpected and disparate directions.

The first years after electrification brought extensive experimentation in terms of both sound generation (i.e. the search for new sounds) and performance interface. The latter trope is perhaps best exemplified by the non-contact interface of the theremin. However, the first signs of conservatism can also be seen in the continuous ring controller that augments the otherwise conventional keyboard of the Ondes Martenot (Hunt, 1999). By the late 1920s and early 1930s progress in interface development had stalled and many designers had returned to the familiar keyboard. This can perhaps be related to Risset's (1978) notion of "sociological weight", or the way in which an instrument could become so culturally entrenched as to represent a barrier to its further evolution. Timbral limitations also meant that, after the rapid technological advancements of World War Two, composers instead turned to the expanded sonic possibilities of the electronic/electroacoustic (i.e. tape) music studio. Such studios spread quickly from notable centres in France and Germany to the United States, Japan and beyond. Equipment was not usually intended for musical purposes but instead repurposed from electronic test applications (Manning, 2004; Andrews \& Maloney, 2015). This was a laborious way to create music: the time and effort needed to edit together hundreds of tiny pieces of tape to create structures lasting only a few seconds made it difficult to produce longer pieces, and entirely ruled out their use in live performance. However, the ability to connect (or patch) these impulse generators, filters, amplifiers and other units together in a multitude of configurations (i.e. a simple kind of programming) paved the way for the modular synthesizer.

The newly invented transistor offered significant advantages over vacuum tubes in terms of miniaturisation and robustness. This new technology enabled Harald Bode to create the Audio System Synthesizer in 1960, arguably the first true modular synthesizer (Bode, 1961). In this context, modularity relates to a system divided into a set of units that can be composed to fulfil a larger function. In many cases the same set of units can also be quickly re-composed to perform (sometimes completely) different functions. Crucially, Bode implemented a system of voltage control. This meant that, in theory at least, any module could be connected to and control any other, and thus input need not be limited to the keyboard or other conventional performance interface, but could instead come from any sound or voltage source.

These ideas were influential on and extended by Robert Moog. His Moog Modular (1964) was aimed primarily at professional musicians and keyboard players in particular. However, while the makeup and arrangement of a Moog Modular system could to some extent be specified by the customer, the tried and tested organ-style keyboard was the assumed performance interface. Also, while its patchability suggested flexibility, the design of the Moog favoured the adoption of a subtractive synthesis paradigm, not least because of the distinctive character of its resonant low-pass filter.

Working contemporaneously to Moog but on the opposite coast of the United States, the modular synthesizer developed by Donald Buchla assumed a fundamentally different philosophy. Initially commissioned and informed by the composers Morton Subotnick, Pauline Oliveiros and Ramon Sender of the San Francisco Tape Music Center, Buchla conceived of the 100 series Modular Electronic Music System as a kind of experimental sound laboratory rather than a musical instrument. A relatively conventional keyboard was available in the form of the Model 112 Touch Controlled Voltage Source. This featured twelve keys, each with two preselected voltages, a third voltage proportional to finger pressure, and a fourth pulse output activated if a key was pressed). However, the Model 114 Touch Controlled Voltage Source was also offered. This featured ten independently tuneable, touch-activated keys, each with a corresponding control voltage output and pulse output. That each plate could be tuned finely and independently made it possible to play microtonal, non-Western, or non-standard scales. A form of simple sequencer was also provided in the form of the Model 123 Sequential Voltage Source. Stages could be switched by applying a pulse, usually from a Model 140 Pulse Generator, but also from the pulse output of the Model 112 or 114 touch plates (Dunn, 1992).

Like some other synthesizers of the period (e.g. the ARP 2600), the 100 series featured a dedicated control voltage processor. Derived from circuits found in general purpose analogue computers (typically used in engineering applications), the Model 156 Control Voltage Processor was capable of simple mixing, scaling, and inverting operations. This general-purpose analogue computing heritage was made even more explicit in the Buchla 200 series Electric Music Box of 1970 and the Model 257 Dual Voltage Processor in particular. Each half of the model 257 enabled three voltages to define a single output voltage according to the equation:

$$
V_{a} * K+V_{b} *(1-M)+M * V_{c}+\text { Voffset }=\text { Vout }
$$

This provided a great deal of flexibility in that the same module could mix (albeit two control voltages only), scale, invert, crossfade, and provide a manually adjustable offset, as well as function as a 
voltage-controlled amplifier (VCA) (Verbos, 2008). These possibilities can be explored via the following Pd-extended patch (Dalgleish, 2015).

While the modular synthesizers of Moog and Buchla featured on notable recordings by Wendy Carlos and Morton Subotnick respectively in the late 1960s, they remained prohibitively expensive and inaccessible to all but a few. As Moog realised that many professional musicians were either excluded by the price of the Moog modular or daunted by its complexity, the smaller, cheaper, and more portable Minimoog model $D$ was released in summer 1970. However, the desire for a simpler and more accessible instrument resulted in a loss of flexibility. In contrast to the relatively open-ended programmability of the Moog Modular, the Minimoog featured a much smaller number of options selectable by rocker switches on the front panel. Whereas sales of the Moog Modular had already started to decline by late 1969, the Minimoog was an immediate success. It remained in production until 1981, selling around 40,000 units overall (Holmes, 2013). Its popularity reinforced the connection between the synthesizer and the keyboard in the minds of users and designers alike. Indeed, the two biggest selling synthesizers of all time, the digital Yamaha DX7 (1983) and the Korg MicroKorg (2002) (Synthhead, 2009), adopted a similar portable keyboard-synthesizer paradigm. As analogue keyboard-synthesizers in the 1970s and preset-enabled digital synthesizers (both keyboard and rackmount) in the 1980s took over, the modular synthesizer slid towards obsolescence, leading Milano (1981) to comment that:

\section{[....] the beasts that were the forerunners of today's cut-down, performance-oriented synthesizers have become the exception and not the rule. As pushbutton instruments have become the rule, modular synthesizers have declined in popularity.}

The spread of the personal computer in the 1980s and, particularly, the advent in the 1990s of laptop computers capable of real-time audio processing and sound synthesis, did little to suggest a revival. It is therefore something of an anachronism that the last decade has seen an explosion of renewed interest in modular synthesizers. This includes the growth of the new, Doepfer-created 3U Eurorack format, as well as established players such as Moog re-entering the field. This rapid rise, sudden decline, and unexpected re-emergence (all in about half a century) represents a fundamentally different trajectory to those of most modern acoustic and electric instruments. Moreover, it has caused some paths to be abandoned prematurely, or at least go underexplored, thereby limiting the creative possibilities of the instrument. The success of the more musician-friendly Moog paradigm has meant the Buchla-inspired notion of directly borrowing from general purpose analogue computing is arguably one such path. The influential Wiard synthesizer designer Grant Richter (2008), for instance, has suggested that the contemporary modular synthesizer can be said to utilise only a distinct subset of analogue computers. It is therefore pertinent to consider what might be gained (in terms of possibilities) by revisiting general-purpose analogue computers?

\section{UNTAPPED POSSIBILITIES?}

Analogue computers provide actual rather than virtual representations of model systems. While many different analogue electronic computers were produced, their core functions (or building blocks) are quite consistent. The core functions include:

- addition and subtraction

- multiplication and division

- inversion

- exponention and logarithm

- differentiation and integration

Initially, most of these processes may appear alien to many synthesizer users (Slater, 1998). However, closer inspection reveals that almost all of these processes are possible with more conventional (musical) modular synthesizers. For instance, integration can be described as producing a steadily changing output voltage in response to a constant input voltage. In short, the longer the input is present, the greater the output voltage. However, an integrator can also be described as a type of first-order low-pass filter: something far more familiar to most synthesizer users. Indeed, rather than being unavailable, in many cases existent "musical" terminology obfuscates these functions, or the user interface design hides them or relegates them to secondary importance.

In this paper these "building blocks" provide the basis for an exploratory audiovisual study called Uchronie.

\section{CHION'S AUDIO-VISION}

A theoretical framework for these explorations is provided by Chion's (1994) Audio-Vision: Sound on Screen. The book is divided into two parts. The first part is most relevant here in that it sets out and attempts to define the relationship between sound and image, and how this relationship can affect the experience of the viewer.

The notion of the "audiovisual contract" is particularly pertinent in this regard. Chion (1994) notes that the relationship between sound and image is never "natural" and always an artificial construct, no matter how seamless and convincing. 
He thus describes the audiovisual contract in terms of "a kind of symbolic contract that the audio-viewer enters into, agreeing to think of sound and image as forming a single entity." The believability of this audiovisual construction depends, at least in part, on synchronicity: the apparently meaningful occurrence of sound and image. While the synchronicity of the two is usually planned carefully crafted, incidental or unplanned synchronicity can also occur. Indeed, it may be that people have a natural tendency to actively try to find such links.

Chion (1994) notes however that the audiovisual relationship can go beyond simple synchronicity, and that sound can be used to actively modify or even completely alter the perception of an image. $\mathrm{He}$ uses the term "synchresis" (a fusion of synchronism and synthesis) to describe the potential of re-associating sound and image. Chion (1994) suggests that, for a single image, there are numerous "allowable" sounds, and the exact choice of sound ultimately determines how the visual is read. An example given to support this concept is the automatic door in Star Wars Episode $V$ - The Empire Strikes Back (1980). Studied carefully or seen in slow motion, it is apparent that the visual consists of only two still images: the door closed and the door open. The addition of a sound effect, however, instead creates an audiovisual illusion of the doors smoothly closing. There are numerous examples of similar effects elsewhere. For instance, the fight sequences of a great many Hollywood films rely on sound effects to add a palpable sense of physicality to their carefully choreographed visuals.

\section{RELATED WORK}

The "rediscovery" of analogue electronic computers and their possibilities has been explored by a modest number of researchers in a variety of different fields, most notably within computer science and electrical engineering disciplines. For instance, Ambaum et al. (2011) detail the construction and operation of a small analogue computer designed to aid the exploration of chaotic systems. Elsewhere, the design of a small generalpurpose analogue computer is proposed as a teaching aid; a way of giving electrical engineering students hands on experience with electronic circuits rather than relying solely on circuit simulator software (SDIY, 2010).

A small number of researchers have explored the possibilities of these technologies in a musical context. The most immediately relevant work to this paper is by Slater (1998), who connected a COMDYNA GP-6 analogue computer to a small Buchla modular synthesizer to enable exploration of a range of chaotic equations.
Less directly related, there has been a resurgence of interest in handmade electronic instruments of the kind pioneered in the 1960s by the likes of David Tudor and David Behrman (Collins, 2009). For example, an overtly "low-technology" aesthetic is apparent in the 7-Segment Display instrument developed by the Dirty Electronics ensemble. Initially created for the Athens Video Art festival, the 7-Segment Display is a hand-held instrument that simultaneously generates sound and light from a series of 4-bit 7-segment displays typically found in older digital clocks (Dirty Electronics, 2012).

More generally, the last decade has seen the rise of the Eurorack modular synthesizer: a smaller, and, to some extent, cheaper format than the modular synthesizers of the 1960s and 1970s. Developed as a loose standard by Dieter Doepfer in the mid-1990s, more than 950 modules are now available in the format from 111 different makers (Eurorack Database, 2015). The majority of these generate or process audio or control voltage, although a small number focus on visuals. For instance, Ming Mecca is a modular video game synthesizer created by Bartee (2014).

\section{SYSTEM}

The system used in this paper is a hybrid of Eurorack modular synthesizer (containing both analogue and digital modules) for sound generation and computer-based visualisation. The core elements of this system, and its main interconnections, are outlined in Figure 1.

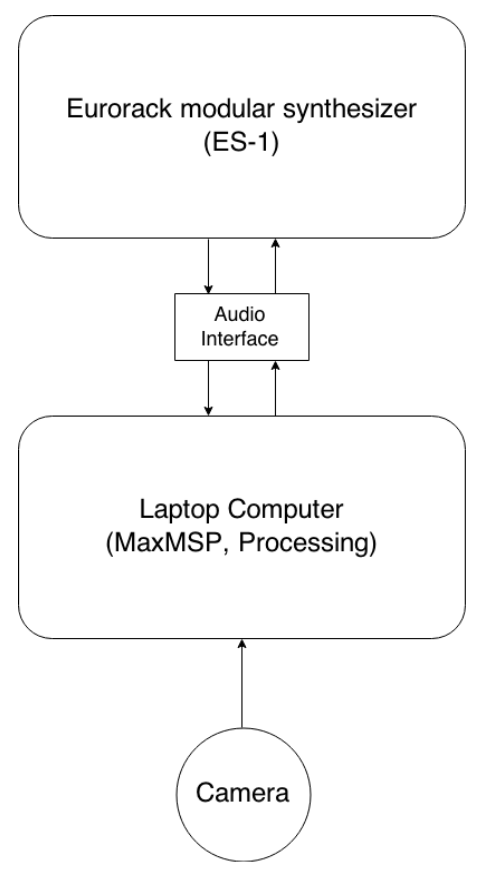

Figure 1. The basic components of the system. 
The $54 \mathrm{U}(18 \times 3 \mathrm{U})$ Eurorack modular synthesizer can be seen below (Figure 2.).

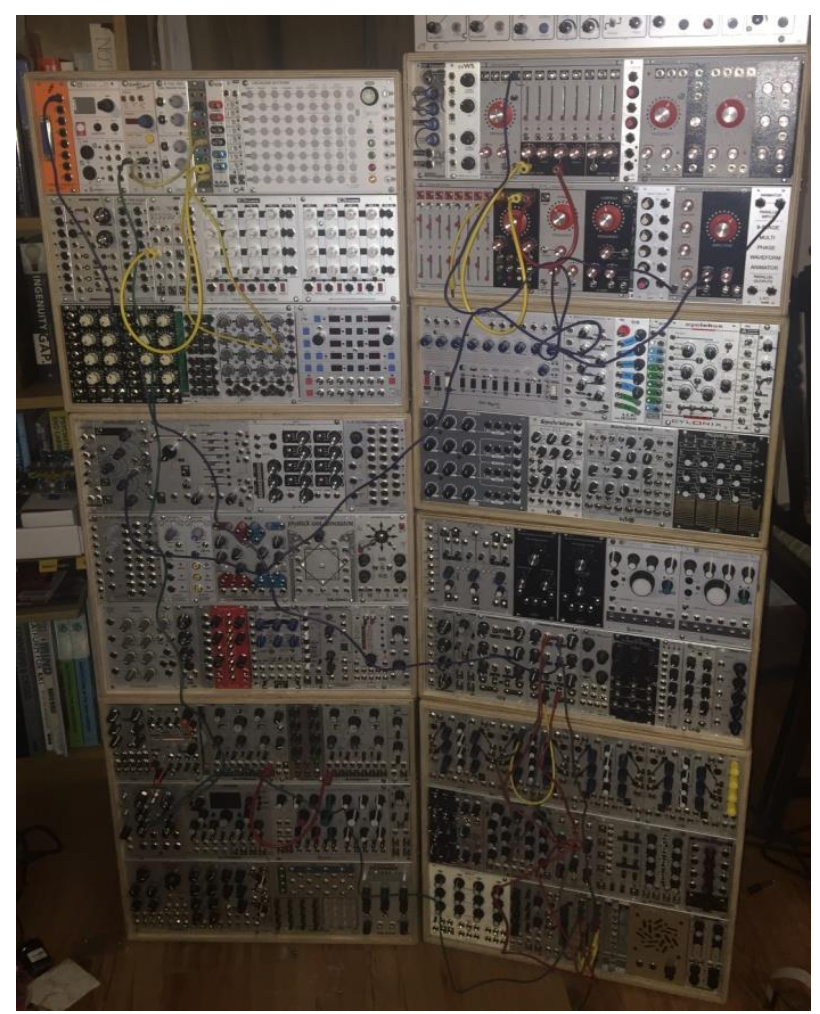

Figure 2. The Eurorack synthesizer used in the project.

Not all of the modules present above are used in this research project. The core modules used are:

- Busy Circuits Pamela's Workout - a programmable clock source with 8 trigger outputs (Busy Circuits, 2013).

- Make Noise Maths - multi-functional module based on the Buchla Model 257 and Serge DUSG. Can act as a 4 channel mixer, slew, envelope generator, LFO, or apply Boolean logic (Make Noise, 2013).

- Steady State Fate Positronic Transient Gate (PTG) - a vactrol-based dual transient generator that can act as an envelope generator, envelope follower, and voltagecontrolled amplifier (VCA) with a maximum gain of 22dB (Steady State Fate, 2014).

- Multiples - enable one signal to be split into multiple copies.

Up to 6 outputs can be tapped simultaneously, sent to the audio interface, and used to provide input to the computer-based visualisation layer. Both MaxMSP/Jitter and the open source Processing are available for the creation of visuals. The audio interface has been internally modified to be DCcoupled, and is therefore able to pass control voltages as well as higher-frequency audio signals.
Finally, the gaze of the user is also tracked in realtime via a small camera. Tracking the gaze in this way can give an indication of the user's attention level and the approximate areas of their focus. In this case, attention level can be piped to the modular synthesizer as a control voltage.

\section{UCHRONIE: AN AUDIOVISUAL STUDY}

Uchronie implements two separate chaotic systems and places them in opposition to each other. The connection between the two is buffered by a simulated mass-spring damper of the kind discussed in Konis (1978).

Inspired by a Doepfer (2004) example, the chaotic systems take as their starting point the following logistic equation:

$$
X_{n}+1=K^{*}\left(X_{n}-X_{n^{2}}\right)
$$

The defining feature of this equation is the selfsimilarity of its output. The practical implementation of the equation on the Eurorack synthesizer can be seen in the rendered image below (Figure 3).

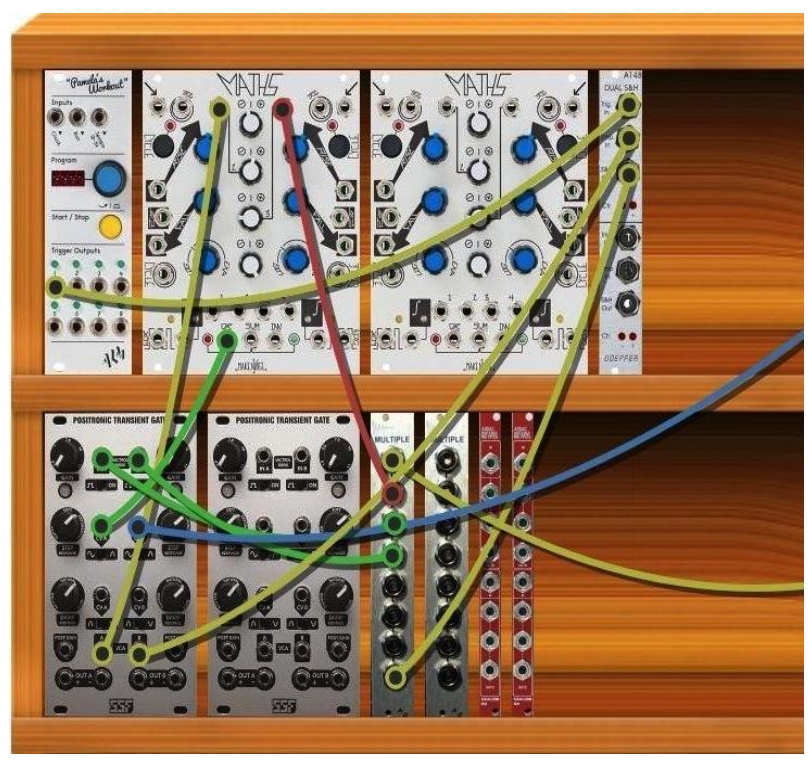

Figure 3. The start of the Uchronie patch.

Note that the two patch cables that extend towards the right side of the image are the CV input for the factor $\mathrm{K}$ (upper right) and the audio output of the chaotic system (lower right). The implementation of the patch on the Eurorack system shown in Figure 2 can be seen below (Figure 4). 


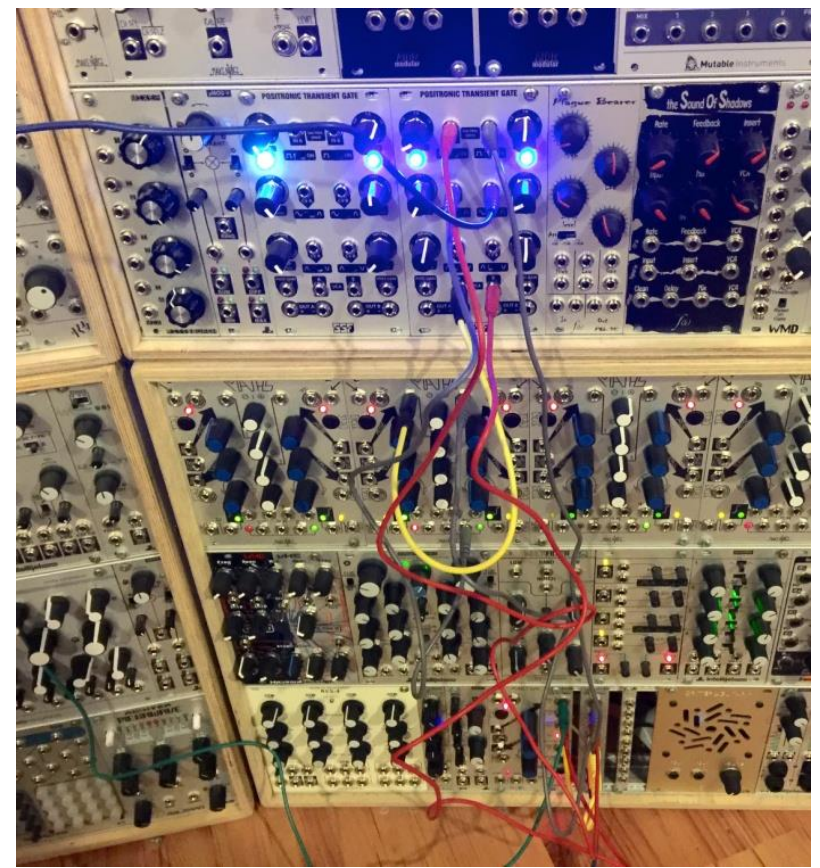

Figure 4. The implementation of the logistic equation on the Eurorack synthesizer.

The key aspects of the patch's construction are as follows:

- a sample and hold $(\mathrm{S} / \mathrm{H})$ is used to sample the last voltage $X_{n}$. The rate at which the $\mathrm{S} / \mathrm{H}$ is triggered determines the overall rate of the system.

- $X_{n^{2}}$ is produced by feeding the output of the $\mathrm{S} / \mathrm{H}$ into the signal and $\mathrm{CV}$ inputs of the left side of a PTG used as a simple VCA.

- the other side of the PTG is used as a second VCA to create the factor $\mathrm{K}$.

- two channels of a Make Noise Maths are used to subtract $X_{n^{2}}$ from $X_{n}$.

The above patch is then duplicated to produce a second chaotic system running in parallel to the first. The topography of the second patch is identical to the first, except that its $\mathrm{S} / \mathrm{H}$ is triggered at a rate that is a multiple of the first clock. The outputs of the two chaotic systems intersect but do not act on each other directly. Instead a simulated mass-spring damper buffers the contact between the two. This is based on the following equations:

$$
\begin{gathered}
F_{m}+F_{d}+F_{s}=0 \\
m \dot{y}+d \dot{y}+s y=0
\end{gathered}
$$

Assuming that is known, the second part can then be rearranged to become:

$$
\ddot{y}=-\frac{1}{m}(d \dot{y}+s y)
$$

From there, $-\dot{y}$ can be generated using an integrator. The force generated by the damper $F_{d}$ can then in turn be generated using the already known value $-\dot{y}$. This is again implemented using a combination of a Make Noise Maths and another PTG. The response of the vactrol in the PTG is tuned to provide the damping. The outputs of the two chaotic systems are then used to drive the ends of the mass-spring damper.

The outputs of the VCAs from both chaotic systems are tapped and used as inputs to the visual system created in MaxMSP/Jitter. The left side of the visual utilises the outputs of the first chaotic system, while the right side of the visual utilises the outputs of the second chaotic system. The visual connection between the two elements is dependent on the output of the simulated spring.

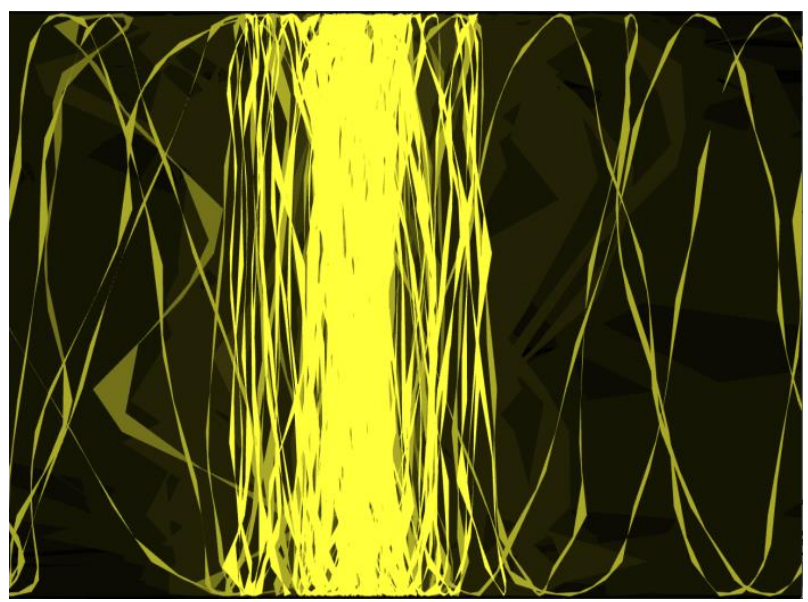

Figure 5. Visuals from Uchronie.

The influence of each of the two chaotic systems on the mass-spring damper can be individually varied. The amount of influence each exerts is determined by the webcam input. When the user's gaze is focused on a single spot (i.e. static), the influence of the two chaotic systems on the damper is equal (i.e. split 50/50). When the gaze is fleeting rather than fixed however, the influence of one (randomly chosen) system is increased at the expense of the other. In addition to this, as the influence of either system increases, the clock multiplier of the second system is also concurrently increased, up to a maximum of $10 \mathrm{X}$ the rate of first system. If the relationship between gaze and the system's response is not obvious, over time it starts to grow more familiar, and the user can influence the relationship between sound and image.

\section{CONCLUSIONS AND FUTURE WORK}

The musical exploration of concepts taken from the histories of analogue computing is not so much a case of creating new systems, as teasing out what is already there, however discreetly hidden. If this may require significant readjustment for someone acclimatised to keyboard synthesizers and other 
conventional electronic instruments, the domain is also interesting from a design perspective. In particular, the absence of stable and controllable pitched sounds means that conventional performance interfaces such as the keyboard are poorly suited. Thus, it may be suggested these kinds of systems call for the development of alternative (i.e. more appropriate) user interfaces, essentially by default. Future work will therefore involve the development of a tangible user interface that is focussed on making the possibilities of the system accessible in real-time performance.

\section{ACKNOWLEDGEMENTS}

With thanks to the Department of Music and Music Technology at the University of Wolverhampton and Luke Bosworth of the Institute for Employment Research at the University of Warwick, UK.

\section{REFERENCES}

Ambaum, M., Harrison, G., Buiting, J., \& Beckers, T. (2011) The chaos machine: analogue computing rediscovered (2). Elektor, 418, 72-75.

Andrews, B. \& Maloney, P. (2015) Bad at Sports Interview with Pauline Oliveros.

http://www.artpractical.com/column/bad-at-sportsinterview-with-pauline-oliveros/ (retrieved 9 March 2015).

Bartee, J. (2014) Ph.D. thesis, Brown University.

Bicknell, S. (1996) The History of the English Organ. Cambridge University Press, Cambridge, 11.

Bode, H. (1961) Sound Synthesizer Creates New Musical Effects. Electronics, 1 December 1961.

Busy Circuits (2013) Pamela's Workout Manual. http://busycircuits.com/docs/alm001-manual.pdf (retrieved 13 March 2015).

Collins, N. (2009) Handmade Electronic Music: The Art of Hardware Hacking. Routledge, New York.

Dalgleish, M. (2015) Buchla 257-style Pd patch. https://drive.google.com/file/d/0B kIPk6lBMZ8MDJ 0OWt0T1F1ak0/view?usp=sharing (retrieved 10 March 2015).

Dirty Electronics (2012) 7-Segment Display Prototype. https://vimeo.com/41902897 (retrieved 11 March 2015).

Doepfer (2004) System A-100 Manual. http://www.doepfer.de/a100 man/A100 Manual co mplete.pdf (retrieved 10 October 2014).

Dunn, D. (1992) Pioneers of Electronic Art. Ars Electronica, Linz, 98-99.
Eurorack Database (2015) Module List. http://www.eurorackdb.com/node/h module list (retrieved 18 March 2015).

Holmes, T. (2013) Noise and Notations. http://thomholmes.com/Noise and Notations/Noise and Notations Blog/Entries/2013/1/14 The Grea test Moog Albums of 1970.html (retrieved 9 March 2015).

Hunt, A. (1999) Radical User Interfaces for Realtime musical control. Ph.D. thesis, University of York, UK.

Johnson, S. (2011) Where Good Ideas Come From: The Natural History of Innovation. Riverhead Books, New York.

Kronis, P. J. (1978) Analogue Computer - Part 4. Practical Electronics, December 1978, 1236-1239.

Lynett, D. \& Quesada, V. (1978) Jean-Claude Risset. Synapse, March/April 1978, 26-32.

Make Noise (2013) Maths Manual. http://www.makenoisemusic.com/manuals/MATHS manual2013.pdf (retrieved 12 March 2015).

Manning, P. (2004) Electronic and Computer Music. Oxford University Press, Oxford.

McKinnon, J. W. (2014) Hydraulis. Oxford Music Online.

http://www.oxfordmusiconline.com/subscriber/articl e/grove/music/13639 (retrieved 11 February 2015).

Meeùs, N. (2014) Keyboard. Oxford Music Online. http://www.oxfordmusiconline.com/subscriber/articl e/grove/music/14944 (retrieved 16 February 2015).

Owen, J. (2009) Bone Flute is Oldest Instrument, Study Says. National Geographic, 24 June 2009. http://news.nationalgeographic.com/news/2009/06/ 090624-bone-flute-oldest-instrument.html (retrieved 9 March 2015).

Paradiso, J. A. (1998) Electronic Music Interfaces: New Ways to Play, IEEE Spectrum (cover article), 34(2), 18-30, December 1997.

Richter, G. (2008) Communication on the Yahoo Wiard Users Group. Yahoo Groups. https://groups.yahoo.com/neo/groups/wiardgroup (retrieved, 12 March 2015).

SDIY (2010) Analogue Computer. SDIY.org. http://www.sdiy.org/pinky/ac/ac.html (retrieved 11 February 2015).

Slater, D. (1998) Chaotic Sound Synthesis. Computer Music Journal, 22(2), 12-19.

Steady State Fate (2014) Official PTG Patchbook. http://static1.squarespace.com/static/5258cf48e4b0 0d699d0ae2de/t/525ef493e4b092595ba1df9d/1381 954707235/OfficialPTGpatchBOOKver1 02.pdf (retrieved 13 March 2015). 
Synthhead (2009) The Korg MicroKorg: The Most Popular Synthesizer Of All Time?

http://www.synthtopia.com/content/2009/05/22/thekorg-microkorg-the-most-popular-synthesizer-of-alltime/ (retrieved 9 March 2015).
Verbos, M. (2008) Musings on Voltage Processors. http://buchlatech.blogspot.co.uk/2008/10/musingson-voltage-processors.html (retrieved 9 March 2015). 